\title{
1. Stabilization by sampled and discrete feedback with positive sampling rate
}

Lars Grüne, Fachbereich Mathematik, J.W. Goethe-Universität, Postfach 1119 32, 60054 Frankfurt am Main, Germany.

\subsection{Introduction}

The problem of static state feedback stabilization of control systems is one of the classical problems in mathematical control theory. Whereas for linear control systems a well known result states that if a system is asymptotically controllable then it also asymptotically stabilizable by a continuous static state feedback (in fact, even by a linear one), this property fails to hold for nonlinear systems. The well known work of Brockett [2] makes this statement mathematically precise, and the recent survey [24] gives a good introduction into the geometrical obstructions to continuous feedback stabilization.

Thus, looking for stabilizing static state feedback laws for many nonlinear systems it is inevitable to consider also discontinuous feedback laws. This, however, causes a number of problems both in the theoretical analysis (due to the possible lack of uniqueness of trajectories) as well as in the practical implementation. A reasonable solution concept for systems controlled by discontinuous feedbacks is the idea of sampling: For a given sequence of increasing times (the "sampling times") one evaluates the feedback law at each of these sampling times and uses the resulting control value as a (constant) control up to the next sampling time. Continuing iteratively, it is not difficult to see that the usual assumptions on the right hand side of the control system indeed guarantee existence and uniqueness for this sampled trajectory. A slightly more specific concept is the notion of discrete feedback introduced in [7]: Here also sampled trajectories are considered, but instead of using arbitrary sequences of sampling times, here the intersampling times are fixed in advance, possibly depending on the state. Thus the resulting closed loop system is essentially equivalent to a discrete time system.

The concept of sampling is known for quite a while and also used in the context of stabilization, see e.g. [11, 12, 22], but only recently it was observed that for general nonlinear systems asymptotic stabilizability by sampled feedback laws is equivalent to asymptotic controllability [4]. However, one has to be careful in the definition of the behaviour of sampled systems: Although 
it is immediate that for each sequence of sampling times we obtain a unique trajectory, the asymptotic behaviour of this trajectory may strongly depend on the choice of the sampling rate (i.e. the maximal time allowed between two discrete sampling times) The general equivalence result mentioned above, for instance, is only true if we consider sampling rates tending to 0 . Thus, it may be interpreted either as a practical stability result for fixed positive sampling rate, or as "real" stability for all possible limit trajectories for vanishing sampling rates. These, however, will in general not be unique.

In the present paper, we discuss recent results on sampled and discrete stability where special emphasis is put on requirements on the sampling rate needed in order to achieve stability of the sampled closed loop system. In particular we formulate the stability properties under consideration always as stability with positive sampling rate, thus describing the system behaviour of individual sampled trajectories rather than limits of trajectories with vanishing sampling rates. Using this approach we attempt to give a suitable mathematical description for implementations of sampled feedback e.g. using some digital controller, in which arbitrary small sampling rates in general will not be realizable. In fact, the investigation of the effect of different sampling rates is interesting not only for discontinuous feedback laws, since in practice also continuous laws are often implemented in a sampled way using digital controllers, and hence essentially the same problems occur.

For general nonlinear systems, a complete characterization of stabilizability with positive sampling rate has not yet been developed. Such a characterization is, however, possible for nonlinear systems with certain homogenity properties, and will be presented and illustrated in this paper.

For simplicity, here we will only deal with global or semi-global phenomena, however, the concepts can be transferred also to the case where stabilizability is only possible from a proper subset of the state space. Concerning the proofs of the results to be presented, instead of giving all the technical details (for which we will refer to the appropriate literature) we restrict ourselves to the main arguments hoping that this allows the reader to get some insight into the problems without being bothered by too much technicalities.

\subsection{Setup and definitions}

We consider nonlinear control systems of the form

$$
\dot{x}(t)=f(x(t), u(t))
$$

where $u(\cdot) \in \mathcal{U}:=\{u: \mathbb{R} \rightarrow U$, measurable and locally essentially bounded $\}$, $U \subseteq \mathbb{R}^{m}, 0 \in U, f: \mathbb{R}^{d} \times U \rightarrow \mathbb{R}^{d}, f(0,0)=0$ and $f$ is supposed to be continuous in both variables and Lipschitz in $x$ for each $u \in U$.

For all $t \geq 0$ for which the (unique) open loop trajectory of (1.1) exists for some initial $x_{0} \in \mathbb{R}^{d}$, some control function $u(\cdot) \in \mathcal{U}$, and initial time $t_{0}=0$ we denote it by $x\left(t, x_{0}, u(\cdot)\right)$. 
In order to characterize asymptotic behaviour at the origin, recall that a function $\alpha:[0, \infty) \rightarrow[0, \infty)$ is called of class $\mathcal{K}$, if it satisfies $\alpha(0)=0$ and is continuous and strictly increasing (and class $\mathcal{K}_{\infty}$ if it is unbounded), and a continuous function $\beta:[0, \infty)^{2} \rightarrow[0, \infty)$ is called of class $\mathcal{K} \mathcal{L}$, if it is of class $\mathcal{K}$ in the first argument and decreasing to zero in the second variable.

Using this definition we are now able to characterize asymptotic controllability.

Definition 1.2.1. System (1.1) is called asymptotically controllable to the origin) if there exists a class $\mathcal{K} \mathcal{L}$ function $\beta$ such that for each $x_{0} \in \mathbb{R}^{d}$ there exists $u_{x_{0}}(\cdot) \in \mathcal{U}$ with

$$
\left\|x\left(t, x_{0}, u_{x_{0}}(\cdot)\right)\right\| \leq \beta\left(\left\|x_{0}\right\|, t\right) \text { for all } t \geq 0,
$$

and it is called asymptotically controllable with finite controls if it is asymptotically controllable and there exists an open set $N \ni 0$ and a constant $C>0$ such that for all $x_{0} \in N$ the control $u_{x_{0}}(\cdot)$ from above can be chosen with $\left\|u_{x_{0}}(\cdot)\right\|_{\infty}<C$.

Note that sometimes the definition of asymptotic controllability already includes finite controls, e.g. in [4, 24]. Here we do not necessarily demand this technical property, since for certain results we can do without it.

An important tool in the stability analysis is the control Lyapunov function as given by the following definition.

Definition 1.2.2. A continuous function $V: \mathbb{R}^{d} \rightarrow[0, \infty)$ is called a control Lyapunov function, if it is positive definite (i.e. $V(0)=0$ iff $V=0$ ), proper (i.e. $V(x) \rightarrow \infty$ as $\|x\| \rightarrow \infty$ ), and there exists a continuous and positive definite function $W: \mathbb{R}^{d} \rightarrow[0, \infty)$ such that for each bounded subset $G \subset \mathbb{R}^{d}$ there exists a compact subset $U_{G} \subset U$ with

$$
\min _{v \in \operatorname{cof}\left(x, U_{G}\right)} D V(x ; v) \leq-W(x) \text { for all } x \in G
$$

Here $D V(x ; v)$ denotes the lower directional derivative

$$
D V(x ; v):=\liminf _{t \searrow 0, v^{\prime} \rightarrow v} \frac{1}{t}\left(V\left(x+t v^{\prime}\right)-V(x)\right),
$$

$f\left(x, U_{G}\right):=\left\{f(x, u) \mid u \in U_{G}\right\}$, and $\operatorname{cof}\left(x, U_{G}\right)$ denotes the convex hull of $f\left(x, U_{G}\right)$.

It is a well known result in control theory that system (1.1) admits a control Lyapunov functions if and only if it is asymptotically controllable with finite controls.

Finally, we introduce the concepts of sampled and discrete feedback control. 
Definition 1.2.3. (i) A sampled feedback law is is a (possibly discontinuous) map $F: \mathbb{R}^{d} \rightarrow U$ with $\sup _{x \in K}\|F(x)\|<\infty$ for all compact $K \subset \mathbb{R}^{d}$ which is applied the following way:

An infinite sequence $\pi=\left(t_{i}\right)_{i \in \mathbb{N}_{0}}$ of times satisfying

$$
0=t_{0}<t_{1}<t_{2}<\ldots \text { and } t_{i} \rightarrow \infty \text { as } i \rightarrow \infty
$$

is called a sampling schedule. The values

$$
t_{i}, \quad \Delta t_{i}:=t_{i+1}-t_{i}, \quad \text { and } \quad d(\pi):=\sup _{i \in \mathbb{N}_{0}} \Delta t_{i}
$$

are called the sampling times, intersampling times, and sampling rate, respectively. For any sampling schedule $\pi$ the corresponding sampled or $\pi$-trajectory $x_{\pi}\left(t, x_{0}, F\right)$ with initial value $x_{0} \in \mathbb{R}^{d}$ at initial time $t_{0}=0$ is defined inductively by

$$
x_{\pi}\left(t, x_{0}, F\right)=x\left(t-t_{i}, x_{i}, F\left(x_{i}\right)\right), \text { for all } t \in\left[t_{i}, t_{i+1}\right], i \in \mathbb{N}_{0}
$$

where $x_{i}=x_{\pi}\left(t_{i}, x_{0}, F\right)$ and $x\left(t, x_{i}, F\left(x_{i}\right)\right)$ denotes the (open loop) trajectory of (1.1) with constant control value $F\left(x_{i}\right)$ and initial value $x_{i}$.

(ii) A discrete feedback law is a sampled feedback law together with a (possibly state dependent) time step $h(x)>0, x \in \mathbb{R}^{d}$ with $\inf _{x \in K} h(x)>0$ for each compact set $K \not \ni 0$, which for each initial value $x_{0} \in \mathbb{R}^{d}$ is applied using sampling schedules $\pi$ satisfying $\Delta t_{i}=h\left(x_{i}\right)$. We denote the corresponding trajectories by $x_{h}\left(t_{i}, x_{0}, F\right)$.

Observe that uniqueness of the $\pi$-trajectories for sampled and discrete feedbacks (on their maximal intervals of existence) follows immediately from the definition also for discontinuous feedback maps $F$.

The sampling schedules specified in the definition of the discrete feedback are uniquely determined by the initial value. The name "discrete feedback" origins from the fact that the resulting sampled closed loop system is in one-to-one correspondence to the discrete time system given by $x_{i+1}=x\left(h\left(x_{i}\right), x_{i}, F\left(x_{i}\right)\right)$. The discrete feedback concept is particularly useful when numerical methods involving discretization of trajectories are used for feedback design, since in this situation the time step $h$ can correspond to some numerical discretization parameter, $\mathrm{cp}$. [7].

\subsection{Stability concepts for sampled systems}

In this section we introduce and discuss appropriate (asymptotic) stability concepts for nonlinear control systems with sampled and discrete feedback. In contrast to the classical case, here we have an additional parameter, namely the sampling rate, which we take into account in our definition. 
Definition 1.3.1. We call the sampled closed loop system from Definition 1.2.3(i)

(i) semi-globally practically stable with positive sampling rate, if there exists a class $\mathcal{K} \mathcal{L}$ function $\beta$ such that for each open set $B \subset \mathbb{R}^{n}$ and each compact set $K \subset \mathbb{R}^{n}$ satisfying $0 \in B \subset K$ there exists $\Delta t>0$ such that

$$
x_{\pi}\left(t, x_{0}, F\right) \notin B \Rightarrow\left\|x_{\pi}\left(t, x_{0}, F\right)\right\| \leq \beta\left(\left\|x_{0}\right\|, t\right)
$$

for all $t \geq 0$, all $x_{0} \in K$ and all $\pi$ with $d(\pi) \leq \Delta t$,

(ii) semi-globally stable with positive sampling rate, if (i) holds and the sampling rate $\Delta t>0$ can be chosen independently of $B$,

(iii) globally practically stable with positive sampling rate if (i) holds and the sampling rate $\Delta t>0$ can be chosen independently of $K$,

(iv) globally stable with positive sampling rate if (i) holds and the sampling rate $\Delta t>0$ can be chosen independently of $K$ and $B$.

We call the stability in (i)-(iv) exponential if the function $\beta$ satisfies $\beta\left(\left\|x_{0}\right\|, t\right) \leq C e^{-\sigma t}\left\|x_{0}\right\|$ for constants $C, \sigma>0$ which may depend on $K$, and uniformly exponential if $C, \sigma>0$ can be chosen independently of $K$.

Note that each of the concepts (ii)-(iv) implies (i) which is exactly the sstability property as defined in [4], cf. also [24, Sections 3.1 and 5.1]. In particular, any of these concepts implies global stability for the (possibly nonunique) limiting trajectories as $h \rightarrow 0$. The difference "only" lies in the performance with positive sampling rate. From the applications point of view, however, this is an important issue, since e.g. for an implementation of a feedback using some digital controller arbitrary small sampling rates in general will not be realizable. Furthermore if the sampling rate tends to zero the resulting stability may be sensitive to measurement errors, if the feedback is based on a non-smooth control Lyapunov function, see [17, 24]. In contrast to this it is quite straightforward to see that for a fixed sampling rate the stability is in fact robust to small errors in the state measurement (small, of course, relative to the norm of the current state of the system) if there exists a corresponding Lipschitz continuous control Lyapunov function, cf. [24, Theorem E].

Analogously, we define the corresponding concepts for systems controlled by discrete feedback.

Definition 1.3.2. We call the discrete feedback controlled system from Definition 1.2.3(ii)

(i) semi-globally practically stable with positive sampling rate, if there exists a class $\mathcal{K} \mathcal{L}$ function $\beta$ such that

$$
\left\|x_{h}\left(t, x_{0}, F\right)\right\| \leq \beta\left(\left\|x_{0}\right\|, t\right)
$$

for all $x_{0} \in \mathbb{R}^{d}$,

(ii) semi-globally stable with positive sampling rate, if (i) holds and the time step $h$ satisfies $\inf _{x \in K} h(x)>0$ for all compact sets $K \subset \mathbb{R}^{d}$,

(iii) globally practically stable with positive sampling rate if (i) holds and 
the time step $h$ satisfies $\inf _{x \notin B} h(x)>0$ for all open sets $B \subset \mathbb{R}^{d}$ with $0 \in B$, (iv) globally stable with positive sampling rate if (i) holds and the time step $h$ satisfies $\inf _{x \in \mathbb{R}^{d}} h(x)>0$.

Again, we call the stability in (i)-(iv) exponential if $\beta$ satisfies $\beta\left(\left\|x_{0}\right\|, t\right) \leq$ $C e^{-\sigma t}\left\|x_{0}\right\|$ for constants $C, \sigma>0$ which may depend on $K$, and uniformly exponential if $C, \sigma>0$ can be chosen independently of $K$.

In fact, it is not difficult to see that the following implications hold.

Proposition 1.3.1. Each of the sampled stability concepts from Definition 1.3.1(i)-(iv) implies the corresponding discrete stability concept from Definition 1.3.2(i)-(iv).

Proof. We show the implication Definition 1.3.1(i) $\Rightarrow$ Definition 1.3.2(i), the other implications follow similarly.

Assume Definition 1.3.1(i) holds for some class $\mathcal{K} \mathcal{L}$ function $\beta$. Consider a sequence of compact sets $\left(K_{i}\right)_{i \in \mathbb{N}}$ with $K_{i} \subset K_{i+1}$ and $\bigcup_{i \in \mathbb{N}} K_{i}=\mathbb{R}^{d}$, and a sequence of open sets $\left(B_{i}\right)_{i \in \mathbb{N}}$ with $B_{i+1} \subset B_{i}$ and $\bigcap_{i \in \mathbb{N}} B_{i}=\{0\}$, such that $B_{1} \subset K_{1}$. For each pair $K_{i}$ and $B_{i}, i \in \mathbb{N}$ denote by $\tau_{i}>0$ the value $\Delta t$ from the assumption. Now for each point $x \in \mathbb{R}^{d}$ we pick the minimal index $i(x) \in \mathbb{N}$ such that $x \in K_{i(x)} \backslash B_{i(x)}$ and define the time step $h$ via $h(x):=\tau_{i(x)}$.

Then from the construction of $h$ and the assumption it follows that

$$
\left\|x_{h}\left(t, x_{0}, F\right)\right\| \leq \beta\left(\left\|x_{0}\right\|, 0\right) \text { for all } t \geq 0 .
$$

Furthermore we can conclude that for each $i \in \mathbb{N}$ there exists times $t_{i}>0$ and $T_{i}>0$ with

$$
x_{h}\left(t, x_{0}, F\right) \in B_{i+1} \text { for all } x_{0} \in B_{i}, t \geq t_{i}
$$

and

$$
x_{h}\left(t, x_{0}, F\right) \in K_{i-1} \text { for all } x_{0} \in K_{i}, t \geq T_{i} .
$$

Using the assumption and these two properties by induction it follows that there exist times $s_{i}>0$ such that

$$
x_{h}\left(t, x_{0}, F\right) \in B_{i} \text { for all } x_{0} \in K_{i} \backslash K_{i-1}, t \geq s_{i} .
$$

which, together with (1.2) implies the existence of the desired class $\mathcal{K} \mathcal{L}$ function (which, however, in general will not coincide with the original $\beta$.)

It is an open question whether the converse implications also hold. The only exception is the case of semi-global practical stability where the following (much stronger) theorem holds, whose main statement goes back to [4].

Theorem 1.3.1. Consider the system (1.1). Then the following properties are equivalent

(i) The system is asymptotically controllable with finite controls 
(ii) There exists a feedback $F$ such that the sampled closed loop system is semi-globally practically stable with positive sampling rate

(iii) There esists a feedback $F$ and a time step $h$ such that the discrete feedback controlled system system is semi-globally practically stable with positive sampling rate

Sketch of Proof. "(ii) $\Rightarrow$ (iii)" follows from Proposition 1.3.1, "(iii) $\Rightarrow$ (i)" is immediately clear.

We sketch the basic idea of the proof of "(i) $\Rightarrow$ (ii)", for a detailled proof see [4]. From [23] asymptotic controllability with finite controls implies the existence of a continuous control Lyapunov function $V_{0}$.

For a positive parameter $\beta>0$ we consider the approximation of $V_{0}$ by the (quadratic) inf-convolution

$$
V_{\beta}(x)=\inf _{y \in \mathbb{R}^{d}}\left\{V_{0}(y)+\frac{\|x-y\|^{2}}{2 \beta^{2}}\right\}
$$

For each $x \in \mathbb{R}^{d}$ we denote by $y_{\beta}(x)$ a point realizing the minimum on the right hand side of this definition, and define

$$
\zeta_{\beta}(x):=\frac{x-y_{\beta}(x)}{2 \beta^{2}} .
$$

Then a straightforward but technical calculation shows that with $F$ defined by

$$
\left\langle\zeta_{\beta}(x), f(x, F(x))\right\rangle=\inf _{u \in U_{G}}\left\langle\zeta_{\beta}(x), f(x, u)\right\rangle
$$

we obtain

$$
V_{\beta}\left(x\left(\tau, x_{0}, F\left(x_{0}\right)\right)-V_{\beta}(x) \leq-\tau W\left(x_{0}\right)+\omega_{\beta}\left(x_{0}\right) \tau+C\left(x_{0}\right) \frac{\tau^{2}}{\beta^{2}}\right.
$$

where $\omega_{\beta}\left(x_{0}\right) \rightarrow 0$ as $\beta \rightarrow 0, \omega_{\beta}$ depends on $\beta$ and on the modulus of continuity of $V$ in $x_{0}$, and $C\left(x_{0}\right)>0$ is a suitable constant essentially depending on $\left|f\left(x_{0}, F\left(x_{0}\right)\right)\right|$ (in fact, behind this estimate lies the theory of proximal sub- and supergradients, see e.g. [3] for an exposition).

By a compactness argument now on each ring $R=\left\{x \in \mathbb{R}^{d} \mid 0<\alpha_{1} \leq\right.$ $\left.\|x\| \leq \alpha_{2}\right\}$ we can formulate inequality (1.3) uniformly for $x_{0} \in R$, which for $\beta>0$ and $\tau>0$ sufficiently small implies that on $R$ the function $V_{\beta}$ is a control Lyapunov function which decreases along $x\left(t, x_{0}, F\left(x_{0}\right)\right)$ for $t \in[0, \tau]$. Choosing a growing family of rings $R_{i} \subset R_{i+1}$ covering $\mathbb{R}^{d} \backslash\{0\}$ and carefully (and rather technically) "gluing" the feedback together on $\partial R_{i}$ finally yields the assertion.

This result in fact states that a stabilizing sampled feedback can always be found under the assumption of asymptotic controllabilty, provided we allow vanishing sampling rates. The question we want to address in the remaining sections is whether one can give conditions under which (sampled or discrete) 
stability with some fixed positive sampling rate can be achieved. Looking at the Proof of Theorem 1.3.1, one sees that the regularity of $V$ plays a crucial role in estimate (1.3) (via the function $\omega$ ) and hence in the choice of the time step $\tau$. Thus one might conjecture that certain regularity properties of the corresponding control Lyapunov function could serve as a sufficient condition. However, the example discussed in the next section shows that even the existence of a $C^{\infty}$ control Lapunov function does not necessarily help.

\subsection{A counterexample to stabilizability with positive sampling rate}

In this section we briefly discuss an example where stability by discrete or sampled feedback with positive sampling rate is not possible. Consider the system

$$
\begin{aligned}
& \dot{r}=r(\theta-u)^{2}-r^{2} \\
& \dot{\theta}=1
\end{aligned}
$$

written in polar coordinates $r \in[0, \infty), \theta \in[0,2 \pi)$, with $U=\mathbb{R}$.

Obviously the (classical) feedback $F(r, \theta)=\theta$ stabilizes this system.

However, considering the ball $B_{1}:=\{(\theta, r) \mid \theta \in[0,2 \pi), r \in[0,1)\}$ and fixing some arbitrary $h>0$ it is easily seen that any trajectory with initial value $\left(\theta_{0}, r_{0}\right) \in B_{1}$ which stays in $B_{1}$ for $t \in[0, h]$ satisfies

$$
\left\|r\left(t, r_{0}, u\right)\right\| \geq C_{1} r_{0} \text { for all } u \in U, t \in[0, h]
$$

for suitable some $C_{1}>0$. Moreover, there exist constants $u_{0}>0$ and $C_{2}>0$ such that

$$
\left\|r\left(t, r_{0}, u\right)\right\| \leq C_{2} r_{0} \text { for all }|u|<u_{0}, t \in[0, h]
$$

and

$$
|| r\left(t, r_{0}, u\right)|| \geq r_{0}+t C_{1} r_{0} \text { for all }|u| \geq u_{0}, t \in[0, h] .
$$

Thus for each $u \in U$ with $|u|<u_{0}$ from (1.4) and (1.5) we can conclude

$$
\begin{aligned}
r\left(h,\left(r_{0}, \theta_{0}\right), u\right)-r_{0} & \geq \int_{0}^{h}\left(\theta_{0}+\tau-u\right)^{2} C_{1} r_{0}-C_{2}^{2} r_{0}^{2} d \tau \\
& =\left(\left(\theta_{0}-u\right)^{2} h+\left(\theta_{0}-u\right) h^{2}+\frac{h^{3}}{3}\right) C_{1} r_{0}-h C_{2}^{2} r_{0}^{2} \\
& \geq \frac{h^{3}}{12} C_{1} r_{0}-h C_{2}^{2} r_{0}^{2}
\end{aligned}
$$

for all trajectories with $r\left(t,\left(r_{0}, \theta_{0}\right), u\right) \in B_{1}$ for all $t \in[0, h]$ where for the last inequality we used that the minimum in $u \in U$ is attained for $u=h / 2+\theta_{0}$. 
From this estimate and inequality (1.6) we can finally conclude that any sampled closed loop trajectory with intersampling times $\Delta t_{i} \geq h$ with $\left(\theta_{0}, r_{0}\right) \in B_{\varepsilon}(0):=\{(\theta, r) \mid \theta \in[0,2 \pi), r \in[0, \varepsilon)\}$ leaves $B_{\varepsilon}(0)$ in finite time for each $\varepsilon<\min \left\{1, C_{1} h^{2} /\left(12 C_{2}^{2}\right)\right\}$, and consequently neither sampled nor discrete stability with positive sampling rate are possible.

We finally note that the function $V(r, \theta)=r^{2}$ is a $C^{\infty}$ control Lyapunov function for this system, and that the vector field is $C^{\infty}$, hence these regularity properties do not imply stabilizability with positive sampling rate.

\subsection{Homogeneous systems}

In this section we summarize results from [10] which show that for homogeneous systems the stabilizability properties with positive sampling rate can be fully determined just by looking at the degree of the system. Stabilization of homogeneous systems has already been investigated by a number of authors, see e.g. [14, 15, 16, 19, 20, 21, 25].

Let us start by defining what we mean by a "homogeneous system". Here we slightly relax the Lipschitz condition on the vector field $f$ and do only assume Lipschitz continuity in $x \in \mathbb{R}^{d} \backslash\{0\}$.

Definition 1.5.1. We call system (1.1) homogeneous if there exist $r_{i}>0$, $i=1, \ldots, d, s_{j}>0, j=1, \ldots, m$ and $\tau \in\left(-\min _{i} r_{i}, \infty\right)$ such that

$$
f\left(\Lambda_{\alpha} x, \Delta_{\alpha} u\right)=\alpha^{\tau} \Lambda_{\alpha} f(x, u) \text { for all } u \in U, \alpha \geq 0
$$

and $\left\{\Delta_{\alpha} u \mid u \in U\right\} \subset U$ for all $\alpha>0$.

For compact $U \subset \mathbb{R}^{m}$ we call system (1.1) homogeneous-in-the-state if there exist $r_{i}>0, i=1, \ldots, d$ and $\tau \in\left(-\min _{i} r_{i}, \infty\right)$ such that

$$
f\left(\Lambda_{\alpha} x, u\right)=\alpha^{\tau} \Lambda_{\alpha} f(x, u) \text { for all } u \in U, \alpha \geq 0
$$

Here

$$
A_{\alpha}=\left(\begin{array}{cccc}
\alpha^{r_{1}} & 0 & \cdots & 0 \\
0 & \ddots & \ddots & \vdots \\
\vdots & \ddots & \ddots & 0 \\
0 & \ldots & 0 & \alpha^{r_{d}}
\end{array}\right) \quad \text { and } \quad \Delta_{\alpha}=\left(\begin{array}{cccc}
\alpha^{s_{1}} & 0 & \cdots & 0 \\
0 & \ddots & \ddots & \vdots \\
\vdots & \ddots & \ddots & 0 \\
0 & \ldots & 0 & \alpha^{s_{m}}
\end{array}\right)
$$

are called dilation matrices. With $k=\min _{i} r_{i}$ we denote the minimal power (of the state dilation) and the value $\tau \in(-k, \infty)$ is called the degree of the system.

The core idea for the construction the stabilizing feedback here lies in finding a homogeneous control Lyapunov function in order to apply the construction of the proof of Theorem 1.3.1. This will first be accomplished for systems homogeneous-in-the-state with a very simple structure, using similar ideas as utilized for semilinear systems in [7, 8, 9]. Assume 


$$
f(\alpha x, u)=\alpha f(x, u) \text { for all } \alpha>0, u \in U
$$

In the notation of Definition 1.5.1 this system is homogeneous-in-the-state with degree $\tau=0$ with respect to the so-called standard dilation $\Lambda_{\alpha}=\alpha I$. We assume furthermore that $U \subset \mathbb{R}^{m}$ is compact. Defining the exponential growth rates

$$
\lambda^{t}\left(x_{0}, u(\cdot)\right):=\frac{1}{t} \ln \frac{\left\|x\left(t, x_{0}, u(\cdot)\right)\right\|}{\left\|x_{0}\right\|}
$$

for each $x_{0} \neq 0$ and each $u(\cdot) \in \mathcal{U}$ it is easily seen from the homogenity property that the system is asymptotically controllable if and only if there exist $T, \sigma>0$ such that for each $x_{0} \neq 0$ there exists $u_{x_{0}}(\cdot) \in \mathcal{U}$ with

$$
\lambda^{t}\left(x_{0}, u_{x_{0}}(\cdot)\right) \leq-\sigma<0
$$

for all $x_{0} \neq 0$ and all all $t \geq T$, cp. [10, Propositions 3.2 and 3.3]. (The idea of considering exponential growth rates is strongly connected with - and in fact inspired by - the spectral theory developed in $[5,6]$.)

Another easy consequence of this homogenity property is the fact that the projection

$$
s\left(t, s_{0}, u(\cdot)\right):=\frac{x\left(t, x_{0}, u(\cdot)\right)}{\left\|x\left(t, x_{0}, u(\cdot)\right)\right\|}, \quad s_{0}=\frac{x_{0}}{\left\|x_{0}\right\|}
$$

of (1.9) onto the unit sphere $\mathbb{S}^{d-1}$ is well defined. A simple application of the chain rule shows that $s$ is the solution of

$$
\dot{s}(t)=f_{\mathbb{S}}(s(t), u(t)), \quad f_{\mathbb{S}}(s, u)=f(s, u)-\langle s, f(s, u)\rangle s
$$

and that for $s_{0}=x_{0} /\left\|x_{0}\right\|$ the exponential growth rate $\lambda^{t}$ satisfies

$$
\lambda^{t}\left(x_{0}, u(\cdot)\right)=\lambda^{t}\left(s_{0}, u(\cdot)\right)=\frac{1}{t} \int_{0}^{t} q\left(s\left(\tau, s_{0}, u(\cdot)\right), u(\tau)\right) d \tau
$$

with $q(s, u)=\langle s, f(s, u)\rangle$. Thus defining the discounted integral

$$
J_{\delta}\left(s_{0}, u(\cdot)\right):=\int_{0}^{\infty} e^{-\delta \tau} q\left(s\left(\tau, s_{0}, u(\cdot)\right), u(\tau)\right) d \tau
$$

and the corresponding optimal value function

$$
v_{\delta}\left(s_{0}\right):=\inf _{u(\cdot) \in \mathcal{U}} J_{\delta}\left(s_{0}, u(\cdot)\right)
$$

from (1.10) and [9, Lemma 3.5(ii)] we obtain that if system (1.9) is asymptotically controllable then for each $\rho \in(0, \sigma)$ there exists $\delta_{\rho}>0$ such that for all $\delta \in\left(0, \delta_{\rho}\right]$ and all $s_{0} \in \mathbb{S}^{n-1}$ the inequality

$$
\delta v_{\delta}\left(s_{0}\right)<-\rho
$$

holds. Note that $v_{\delta}$ is Hölder continuous and bounded for each $\delta>0$, cp. e.g. [1]. We now fix some $\rho \in(0, \sigma)$ and some $\delta \in\left(0, \delta_{\rho}\right]$ and define

$$
V_{0}(x):=e^{2 v_{\delta}(x /\|x\|)}\|x\|^{2} .
$$


Using Bellman's Optimality Principle a straightforward (but tedious) computation shows that the function $V_{0}$ is a control Lyapunov function which is homogeneous with degree $\tau=1$ with respect to the standard dilation and satisfies

$$
\min _{v \in \operatorname{co} f(x, U)} D V_{0}(x ; v) \leq-2 \rho V_{0}(x)
$$

cp. [10, Lemma 4.1].

Now we use this function as the starting point in the proof of Theorem 1.3.1, and proceed analogously (for details see [10, Proposition 4.2]). Note that $V_{\beta}$ inherits the homogenity properties of $V_{0}$, thus $F$ can be chosen to be constant on rays of the form $\alpha x, \alpha>0, x \in \mathbb{R}^{d}$. Now we chose a ring $R$ containing $\mathbb{S}^{d-1}$ and consider inequality (1.3) (with $W(x)=2 \rho V_{0}(x)$ ). Again by a compactness argument, from this inequality we obtain

$$
V_{\beta}\left(x\left(\tau, x_{0}, F\left(x_{0}\right)\right)-V_{\beta}\left(x_{0}\right) \leq-\tau \rho V_{0}\left(x_{0}\right)\right.
$$

for some $\beta>0$ and some $\tau_{0}>0$ sufficiently small, all $\tau \in\left[0, \tau_{0}\right]$ and all $x_{0} \in \mathbb{S}^{d-1}$. Then homogenity immediately implies this inequality for all $x_{0} \in$ $\mathbb{R}^{d}$ and hence the resulting feedback law globally stabilizes system (1.9) with positive sampling rate, in fact even uniformly exponentially.

This result can be carried over to the general homogeneous systems from Definition 1.5.1, leading to the following theorem. Here the function $N(x)$ is given by

$$
N(x):=\left(\sum_{i=1}^{d} x_{i}^{\frac{p}{p_{i}}}\right)^{\frac{1}{p}}
$$

with $p=2 \prod_{i=1}^{d} r_{i}$.

Theorem 1.5.1. Consider a homogeneous system according to Definition 1.5.1 with dilation matrices $\Lambda_{\alpha}$ and $\Delta_{\alpha}$, minimal power $k>0$, and degree $\tau \in(-k, \infty)$, and assume asymptotic controllability.

Then there exists a feedback law $F: \mathbb{R}^{d} \rightarrow U$ satisfying $F(x) \in \Delta_{N(x)} U_{0}$ for some compact $U_{0} \subset U$ and $F\left(\Lambda_{\alpha} x\right)=\Delta_{\alpha} F(x)$ for all $x \in \mathbb{R}^{d}$ and all $\alpha \geq 0$ such that the corresponding sampled closed loop system is either

(i) semi-globally stable (if $\tau>0$ ), or

(ii) globally uniformly exponentially stable (if $\tau=0$ ), or

(iii) globally practically exponentially stable (if $\tau<0$ )

with fixed sampling rate.

The analogous result holds for systems homogeneous-in-the-state; here $F$ satisfies $F(x) \in U$ and $F\left(\Lambda_{\alpha} x\right)=F(x)$ for all $x \in \mathbb{R}^{d}$ and all $\alpha \geq 0$.

Sketch of Proof. (See [10, Theorem 2.6 and 4.3] for a detailled proof.) First observe that the function $N$ satisfies $N\left(\Lambda_{\alpha} x\right)=\alpha N(x)$. Hence if for a homogeneous system we replace $f$ by $f\left(x, \Delta_{N(x)} u\right)$ we obtain a system homogeneous-in-the-state. A straightforward application of the homogenity 
yields that this system is asymptotically controllable with control values in some compact set $U_{0} \subset U$ if and only if the original homogeneous system is asymptotically controllable, see [10, Proposition 6.1]; conversely if $F$ stabilizes the system homogeneous-in-the-state then $\Delta_{N(x)} F(x)$ stabilizes the original homogeneous system. Hence it suffices to show the theorem for systems homogeneous-in-the-state.

To this end consider the manifold $N^{-1}(1):=\left\{x \in \mathbb{R}^{d} \mid N(x)=1\right\}$. Obviously the function $S(x)=x /\|x\|$ gives a diffeomorphism from $N^{-1}(1)$ to $\mathbb{S}^{d-1}$. Thus the function $\Psi(x)=N(x)^{k} S(P(x))$ with $P(x)=\Lambda_{N(x)}^{-1} x$ is a continuous cordinate transformation with continuous inverse (both are also differentiable except possibly at the origin), and replacing $f(x, u)$ by $D \Psi\left(\Psi^{-1}(x)\right) f\left(\Psi^{-1}(x), u\right)$ we obtain a system which is homogeneous in the state with respect to the standard dilation and with degree $\gamma=\tau / k$. Replacing further $f(x, u)$ by $f(x, u)|| x \|^{-\gamma}$ — i.e. applying a time transformation - we end up with a system of type (1.9) for which the stabilizing feedback based on the control Lyapunov function $V_{\beta}$ has been constructed above. Re-translating this to the general system we first have to remove the time transformation which essentially depends on the sign of degree of the system. This affects the sampling rates and thus leads to the three different cases (i), (ii) and (iii). Since the space transformation does not affect the stability properties of the sampled closed loop system we obtain the assertion.

Note that the numerical methods from [7] are easily transferred to the homogeneous case, thus they give a possibility to compute stabilizing discrete feedbacks numerically. See the next section for examples.

Observe that the stabilizing homogeneous feedback corresponds to a homogeneous control Lyapunov function obtained by applying the coordinate transformation $\Psi^{-1}$ to $V_{\beta}$. This may be used to transfer these results to local results for systems approximated by homogeneous systems, similar to $[13,16,18]$.

Furthermore, note that even if a homogeneous system admits a stabilizing continuous static state feedback law, a stabilizing continuous and homogeneous static state feedback for does not exist in general, cp. [21]. One way to overcome the non-homogenity is by using dynamic feedbacks, see [14], the above theorem in fact shows that discontinuous feedbacks provide another way.

If we assume Lipschitz continuity of the homogeneous system in the orogin we immediately obtain $\tau \geq 0$, and thus at least semi-global stabilizability. If we assume global Lipschitz continuity (i.e. the existence of a global Lipschitz constant) this implies $\tau=0$ and thus even global stabilizability. 


\subsection{Examples}

Let us now illustrate our results by two examples. The first example, given by the vector field

$$
f(x, u)=\left(\begin{array}{l}
x_{1}+u \\
3 x_{2}+x_{1} u^{2}
\end{array}\right)
$$

for $x=\left(x_{1}, x_{2}\right)^{T} \in \mathbb{R}^{2}, u \in U=\mathbb{R}$, is taken from [21] where it has been shown that a stabilizing continuous and homogeneous feedback law cannot exist for this system. The vector field $f$ is homogeneous with $\Lambda_{\alpha}=\operatorname{diag}\left(\alpha, \alpha^{3}\right)$ and

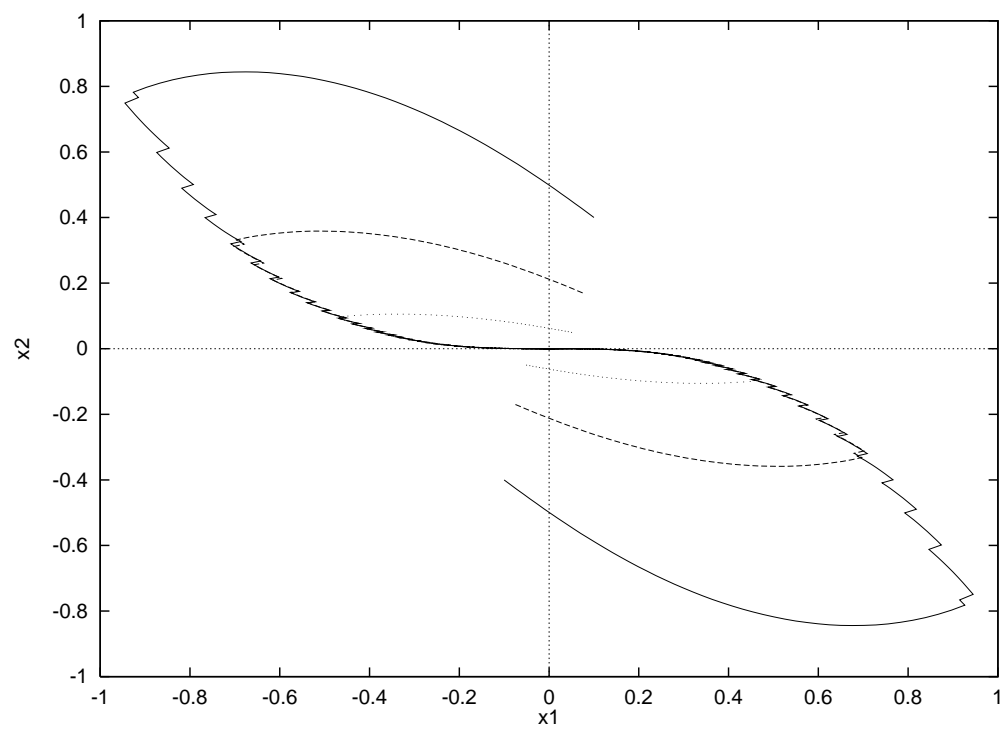

Fig. 1.1. Trajectories for stabilized system (1.11)

$\Delta_{\alpha}=\alpha$. Thus we obtain $N(x)=\left(x_{1}^{6}+x_{2}^{2}\right)^{1 / 6}$. For system (1.11) a stabilizing discrete feedback has been computed numerically using the techniques from [7] extended to the general homogeneous case. Analyzing the switching curves of the numerical feedback in this case it was easy to derive the feedback

$$
F(x)=\left\{\begin{array}{r}
N(x), x_{1} \leq-x_{2}^{3} \\
-N(x), x_{1}>-x_{2}^{3}
\end{array}\right.
$$

stabilizing the sampled system for all sufficiently small sampling rates. Figure 1.1 shows the corresponding (numerically simulated) sampled trajectories for some initial values, here the intersampling times have been chosen as $\Delta t_{i}=0.01$ for all $i \in \mathbb{N}_{0}$.

The second example is the nonholonomic integrator given by Brockett [2] as an example for a system being asymptotically null controllable but not 
stabilizable by a continuous feedback law. In suitable coordinates (cf. [24], where also the physical meaning is discussed) it is given by the vector field

$$
f(x, u)=\left(\begin{array}{r}
u_{1} \\
u_{2} \\
x_{1} u_{2}
\end{array}\right)
$$

for $x=\left(x_{1}, x_{2}, x_{3}\right)^{T} \in \mathbb{R}^{3}, u=\left(u_{1}, u_{2}\right)^{T} \in U=\mathbb{R}^{2}$. For this $f$ we obtain homogenity with $\Lambda_{\alpha}=\operatorname{diag}\left(\alpha, \alpha, \alpha^{2}\right)$ and $\Delta_{\alpha}=\operatorname{diag}(\alpha, \alpha)$, hence $N(x)=$ $\left(x_{1}^{4}+x_{2}^{4}+x_{3}^{2}\right)^{1 / 4}$. Again a stabilizing discrete feedback law has been computed numerically.

Also in this example it should be possible to derive an explicit formula from the numerical results. This is, however, considerably more complicated, since a number of switching surfaces have to be identified. Hence we directly used the numerically computed feedback for the simulation shown in the Figures 1.2-1.4 in different projections; the time step is $h \equiv 0.01$, the controlvalues were chosen as $U_{0}=\{-1,1\}$.

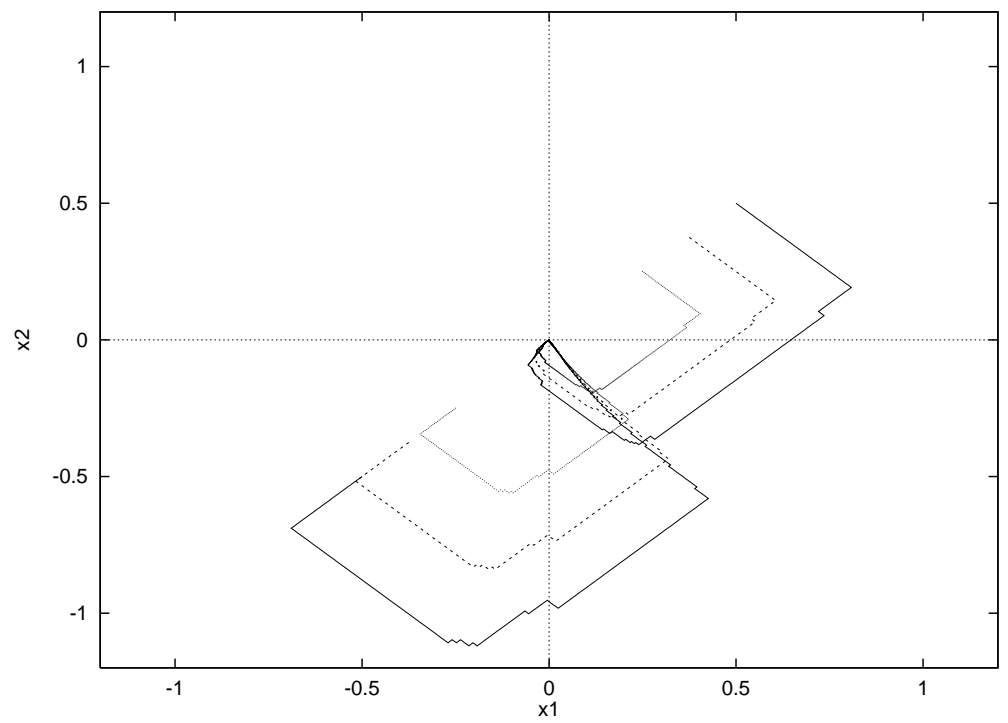

Fig. 1.2. Trajectories for stabilized system (1.12), projected to the $\left(x_{1}, x_{2}\right)$ plane 


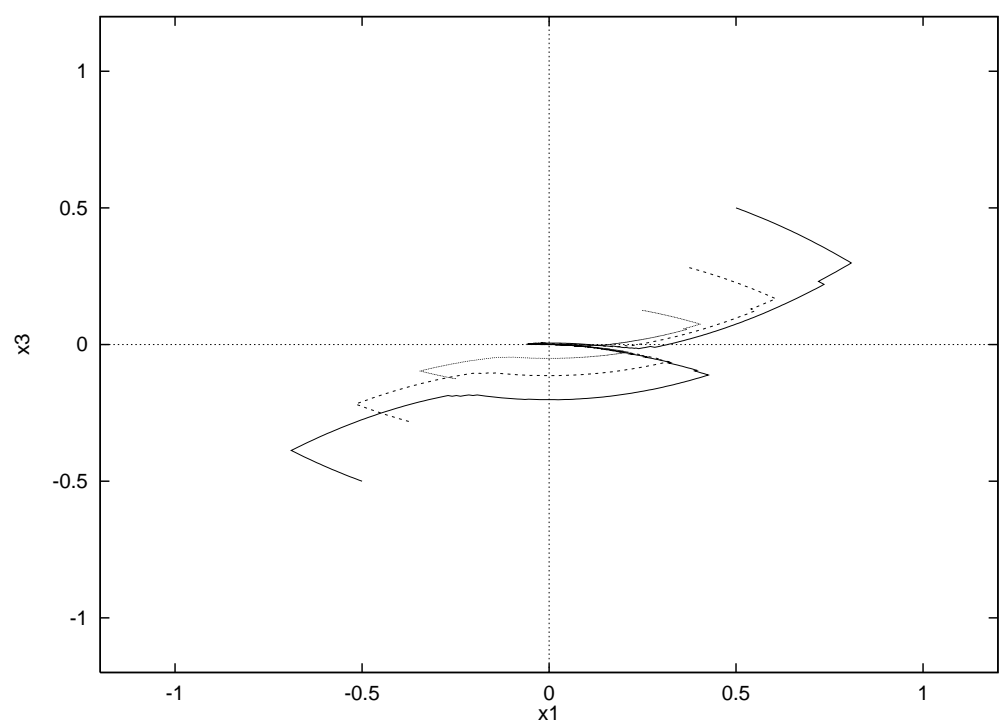

Fig. 1.3. Trajectories for stabilized system (1.12), projected to the $\left(x_{1}, x_{3}\right)$ plane

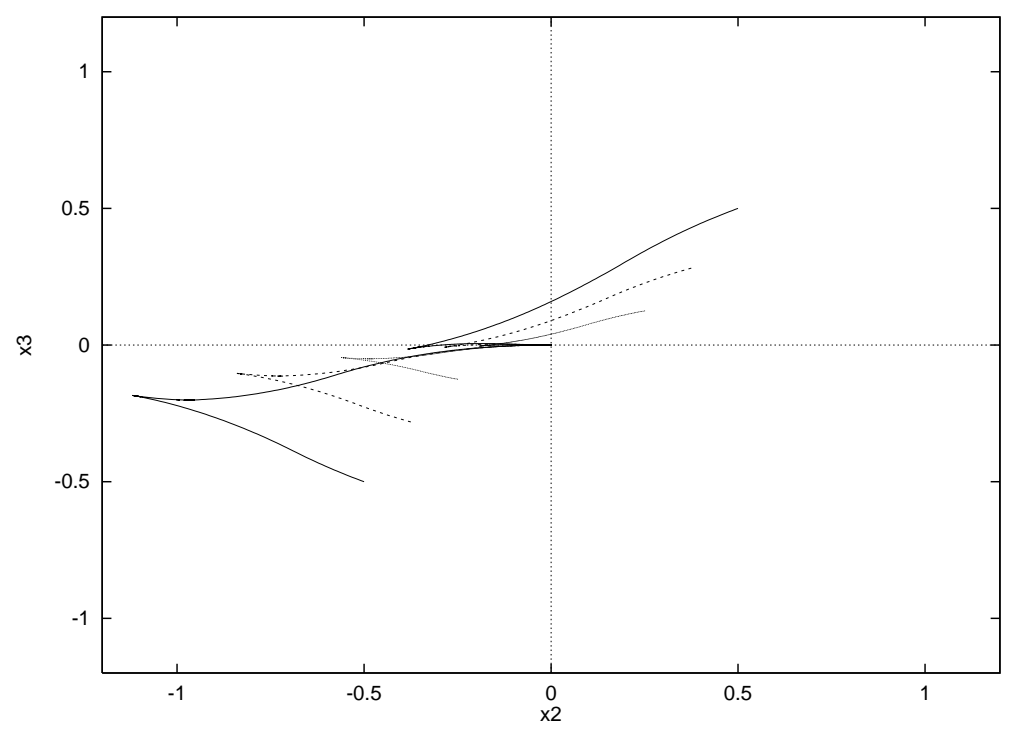

Fig. 1.4. Trajectories for stabilized system (1.12), projected to the $\left(x_{2}, x_{3}\right)$ plane

\section{Summary}

In this paper we discussed the stabilization of systems with sampled and discrete feedback. Whereas this is always possible provided the system un- 
der consideration is asymptotically controllable, in general it can only be achieved by using vanishing intersampling times close to the origin, or far away from it. This fact is illustrated by an example. For general vector fields conditions ensuring sampled or discrete stabilizability with positive sampling rate are still unknown. For homogeneous systems, however, this property can be completely characterized by the degree of homogenity of the system. Two examples of stabilized homogeneous systems illustrate this fact.

Acknowledgement. Parts of this paper have been written while the author was visiting the Dipartimento di Matematica of the Universitá di Roma "La Sapienza", Italy, supported by DFG-Grant GR1569/2-1. 


\section{References}

1. M. Bardi and I. Capuzzo Dolcetta, Optimal Control and Viscosity Solutions of Hamilton-Jacobi-Bellman equations, Birkhäuser, Boston, 1997.

2. R. Brockett, Asymptotic stability and feedback stabilization, in Differential Geometric Control Theory, R. Brockett, R. Millman, and H. Sussmann, eds., Birkhäuser, Boston, 1983, pp. 181-191.

3. F. Clarke, Methods of Dynamic and Nonsmooth Optimization, vol. 22 of CBMS-NSF Regional Conferences Series in Applied Mathematics, SIAM, Philadelphia, 1989.

4. F. Clarke, Y. Ledyaev, E. Sontag, and A. Subbotin, Asymptotic controllability implies feedback stabilization, IEEE Trans. Autom. Control, 42 (1997), pp. 1394-1407.

5. F. Colonius and W. Kliemann, Maximal and minimal Lyapunov exponents of bilinear control systems, J. Differ. Equations, 101 (1993), pp. 232-275.

6. F. Colonius and W. Kliemann, The Dynamics of Control, Birkhäuser, to appear.

7. L. Grüne, Discrete feedback stabilization of semilinear control systems, ESAIM Control Optim. Calc. Var., 1 (1996), pp. 207-224.

8. L. Grüne, Numerical stabilization of bilinear control systems, SIAM J. Control Optim., 34 (1996), pp. 2024-2050.

9. L. Grüne, Asymptotic controllability and exponential stabilization of nonlinear control systems at singular points, SIAM J. Control Optim., 36 (1998), pp. $1585-1603$.

10. L. Grüne, Homogeneous state feedback stabilization of homogeneous control systems, Preprint, Nonlinear Control Abstracts NCA-9-2-981203. Submitted.

11. H. Hermes, On stabilizing feedback attitude control, J. Optimization Theory Appl., 31 (1980), pp. 373-384.

12. H. Hermes, On the synthesis of stabilizing feedback control via Lie algebraic methods, SIAM J. Control Optim., 18 (1980), pp. 352-361.

13. H. Hermes, Nilpotent and high order approximations of vector field systems, SIAM Rev., 33 (1991), pp. 238-264.

14. H. Hermes, Homogeneous feedback control for homogeneous systems, System \& Control Lett., 24 (1995), pp. 7-11.

15. A. Iggidr and J.-C. Vivalda, Global stabilization of homogeneous polynomial systems, Nonlinear Anal., 18 (1992), pp. 1181-1186.

16. M. Kawski, Homogeneous feedback stabilization, in New Trends in Systems Theory (Genova, 1990), Progr. Systems Control Theory, vol. 7, Birkhäuser, Boston, 1991, pp. 464-471.

17. Y. Ledyaev and E. Sontag, A Lyapunov characterization of robust stabilization, J. Nonlinear Anal. To appear.

18. L. Rosier, Homogeneous Liapunov function for continuous vector fields, System \& Control Lett., 19 (1992), pp. 467-473. 
19. E. Ryan, Universal stabilization of a class of nonlinear systems with homogeneous vector field, System \& Control Lett., 26 (1995), pp. 177-184.

20. R. Sepulchre and D. Ayels, Homogeneous Lyapunov functions and necessary conditions for stabilization, Math. Control Signals Systems, 9 (1996), pp. 34-58.

21. R. Sepulchre and D. Ayels, Stabilizability does not imply homogeneous stabilizability for controllable homogeneous systems, SIAM J. Control Optim., 34 (1996), pp. 1798-1813.

22. E. Sontag, Nonlinear regulation: The piecewise linear approach, IEEE Trans. Autom. Control, AC-26 (1981), pp. 346-358.

23. E. Sontag, A Lyapunov like characterization of asymptotic controllability, SIAM J. Control Optim., 21(1983), pp. 462-471

24. E. Sontag, Stability and stabilization: Discontinuities and the effect of disturbances, in Proc. NATO Advanced Study Institute "Nonlinear Analysis, Differential Equations, and Control" (Montreal, Jul/Aug 1998), Kluwer, 1999.

25. J. Tsinias, Remarks on feedback stabilizability of homogeneous systems, Control Theory Adv. Tech., 6 (1990), pp. 533-542. 